\title{
Visual and laserfiuorescence diagnosis with Vistacam of first permanent molar before and after silanization.
}

\author{
Lilyana Shtereva $^{1}$, Veselina Kondeva ${ }^{2}$
}

1. Assistant professor in the Department of Pediatric Dentistry, Faculty of Dental Medicine, Medical University - Plovdiv, Bulgaria;

2. Associate professor in the Department of Pediatric Dentistry, Faculty of Dental Medicine, Medical University - Plovdiv, Bulgaria;

\begin{abstract}
Caries detection on occlusal surfaces and its perfection are main object of scientific interest in dentistry. The initial assessment of hard tooth tissue is normally visual. Apart from purely visual diagnosis, there are other methods for the detection of caries on occlusal surfaces. Some of the modern and reliable methods for diagnosis are fluorescence-based methods. The case presented if of a six year-old girl R.V. from Plovdiv Bulgaria, whose first permanent molar's occlusal surface was examined by visual diagnosis using the system ICDAS II, and laser-fluorescence with VistaCam iX proof. The enamel was sound, and the tooth was sealed, and monitored after 6 and 12 months. The laser-fluorescence diagnostic showed false positive results for initial caries, which was not found after the sealant removal. The sealant was reapplied. It was concluded that VistaCam is a reliable diagnostic method, a useful addition to visual diagnostics on the ICDAS system. Further studies should be performed for more precise results.
\end{abstract}

Keywords: sealants, laser-fluorescence, visual diagnosis 


\section{Background}

Caries detection on occlusal surfaces and its perfection are main object of scientific interest in dentistry. Numerous diagnostic methods and tools have been developed to provide more precise diagnosis. With the technological development a number of new methods and tools are added to the already existing ones in order to increase the reliability of the results. There is no universal method for caries detection; however the right combination of diagnostic methods and tools, appropriate for the clinical situation could give us most detailed information. The initial assessment of hard tooth tissue is normally visual. The visual inspection of a macroscopic intact occlusal surface has a limited sensitivity. By increasing the experience and specific training of the examiners as well as by the use of more precise criteria for visual diagnostics, it increases the accuracy of the diagnosis and sensitivity of the method. Apart from purely visual diagnosis, there are other methods for the detection of caries on occlusal surfaces. Some of the modern and reliable methods for diagnosis are fluorescence-based methods. It is well known that fluorescence- based methods make use of the phenomenon thatcarious lesions fluoresce more strongly than sound tissues when excited by light at specific wavelengths.(1) Preventive dentistry deals with inhibiting occlusal caries on susceptible tooth surfaces such as pits and fissures. The application of pit and fissure sealants is effective in caries reduction. However, periodic monitoring in the dental office is required to assure the effectiveness of the sealants. This monitoring could be performed only using visual diagnosis, to ensure the reliability of the results fluorescence-based methods could be added.

\section{Case description}

A six year-old girl R.V. from Plovdiv Bulgaria came with her mother to our dental office asking for appropriate measures for caries prevention, since the child already had a few obturations on her primary molars, and belonged to the high risk group of caries development. After dental examination was performed, was taken the decision for application of composite-based dental sealant on the first permanent molars. Prior to examination the occlusal surface was cleaned with brush and water, dried, isolated, and then examined. Visual and laser-fluorescence methods were used during the examination of the occlusal surfaces. For the visual examination the visual classification system International Caries Detection and Assessment System (ICDAS-II) was used. The ICDAS II codes are presented in Table 1. 
Table 1- ICDAS II codes

\begin{tabular}{|l|l|l|l|}
\hline \multicolumn{3}{|c|}{ ICDAS II } \\
\hline Scores & Severity of caries & $\begin{array}{l}\text { Restoration } \\
\text { codes }\end{array}$ & Restorations \\
\hline 0 & Sound tooth & 0 & Not restored or sealed \\
1 & First visual change in enamel & 1 & Sealant (partial) \\
2 & Distinct visual change in enamel & 2 & Sealant (full) \\
3 & Localized enamel breakdown & 3 & Tooth-colored restoration \\
4 & Underlying dark shadow from & 4 & Amalgam restoration \\
& dentin & 5 & Stainless steel crown \\
5 & $\begin{array}{l}\text { Distinct cavity with visible dentin } \\
6\end{array}$ & 6 & Porcelain or gold or PFM \\
& Extensive distinct cavity with & & crown, or veneer, or inlay, \\
97 & visible dentin & 7 & or onlay, etc. \\
96 & Tooth missing because of caries & 8 & Tost or broken restoration \\
& Tooth surface cannot be & & Temporary restoration \\
\hline & examined: surface excluded & & \\
\hline
\end{tabular}

After the visual examination on tooth 16 , code- 00 , meaning-sound tooth, not restored or sealed, was given to the upper first permanent molar. After the visual diagnosis, laser-fluorescence diagnosis with VistaCamiX Proof was conducted. For VistaCamiX Proof inspection we used analysis scale of DBSWIN software. Using the VistaCam iX fluorescence-based camera (FC), images of the occlusal tooth surface were taken. The optical head of the camera was placed on the occlusal surface of the tooth and the control ring was pressed in order to freeze the image taken by the camera. Detailed information on the scales of FC is given in figure 1 (2). 


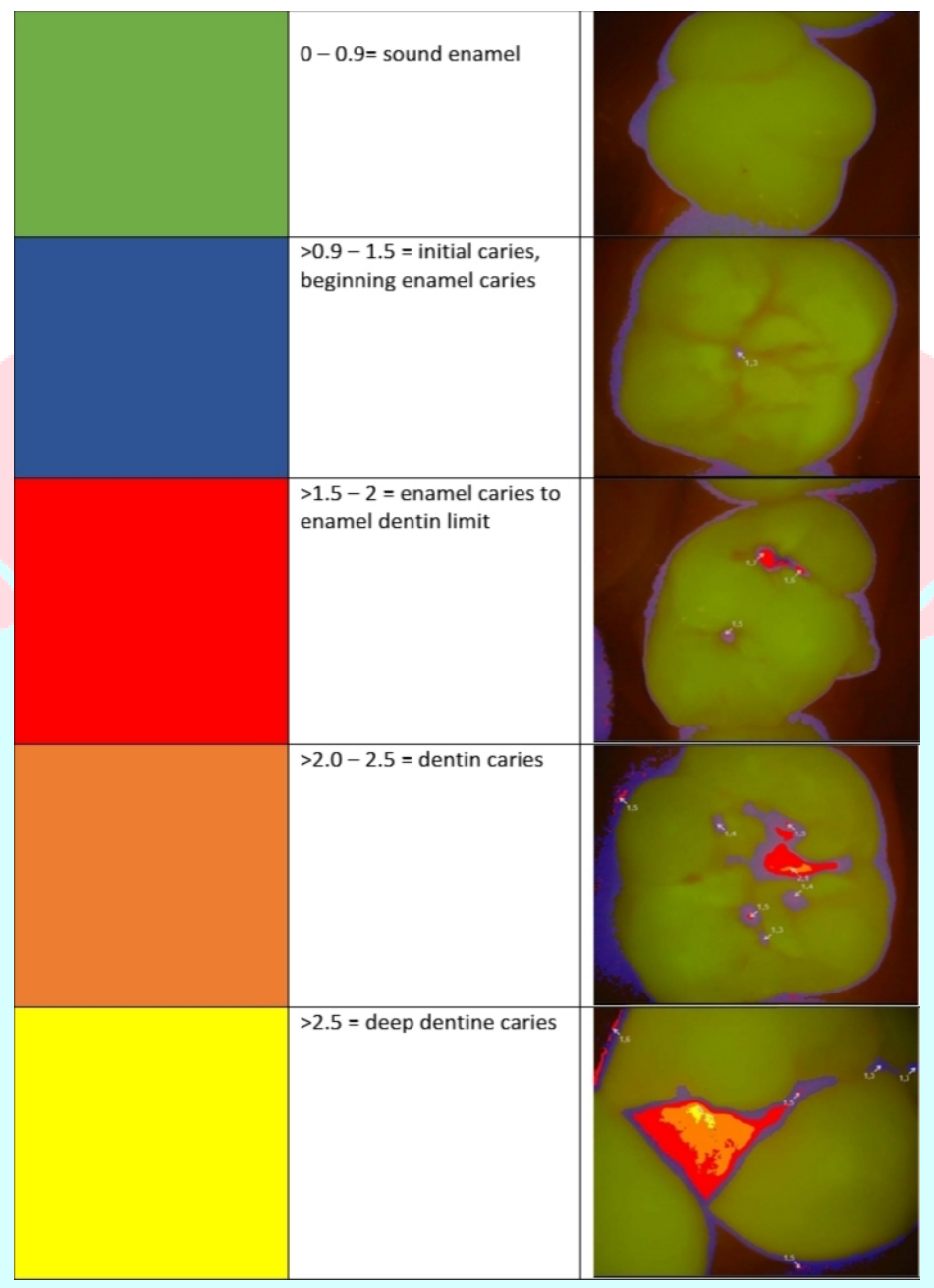

Fig.1- Scales of FC VistaCam iX proof

When examined with VistaCam iX proof only green light typical for sound enamel was emitted (fig 2).

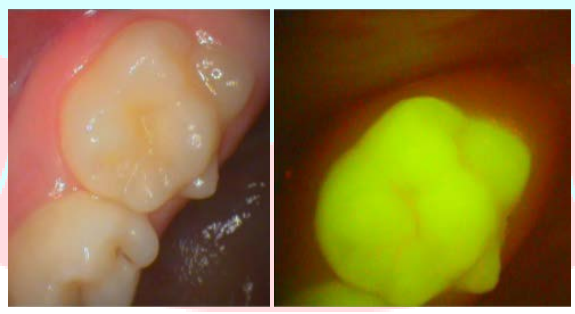

Fig.2- R.V. 6-years old. Visual and LF diagnosis with Vistacam prior to silanisation. 
We proceeded with the silanization. The sealant material used in this case was resin-based sealant -Fissurit F. After cleaning the occlusal surface with low speed handpiece, brush and hydrogen peroxide, the tooth was dried and isolated and sealed, following the instructions of the manufacturer. (fig 3)

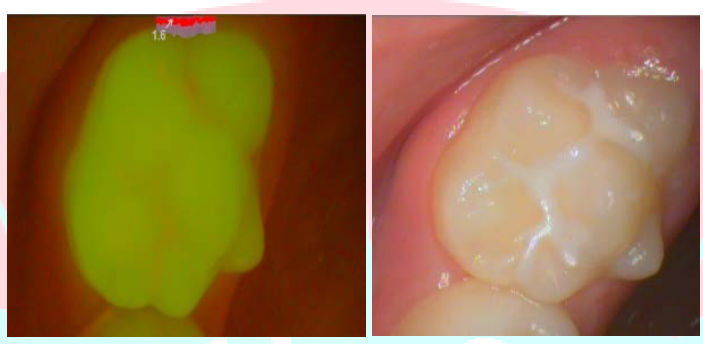

Fig.3- R.V. 6-years old. Visual and LF diagnosis with Vistacam after silanization.

Monitoring was conducted after 6 months, using both methods for diagnosis. There were no changes in the integrity of the sealant, nor any staining or caries: ICDAS II code 20, meaning full sealant and sound tooth. Visual examination was supported by LF diagnostics, performed with VistaCam, which confirmed the result: the occlusal surface fluoresced green. (fig 4)
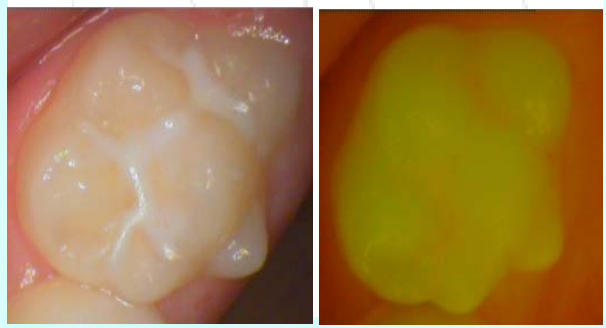

Fig.4- R.V. 6-years old. Visual and LF diagnosis with Vistacam 6 months after silanization.

12 months after the silanization the patient came for a regular check-up. After the visual diagnostics wear of the sealant was established. The code given was 10 - partially remaining sealant and healthy enamel. LF diagnostics with Vistacam was performed. In the image processed by the Vistacam software the changes in the sealant are easily distinguished, as well as small bubble in the sealant was marked in blue. The area with sealant wear is colored in blue-meaning first changes in the enamel. (fig 5) Trough the LF, performed by vistacam, the defect in the sealant was highlighted, which later helped us develop a precise treatment plan. 


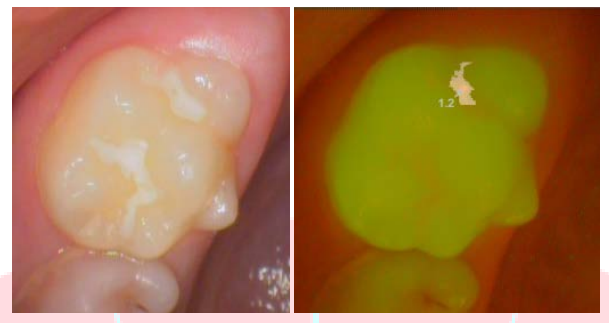

Fig.5- R.V. 6-years old. Visual and LF diagnosis with Vistacam 12 months after silanization.

The sealant was replaces, and new monitoring of the occlusal surface is going to be performed. However, no caries was detected after the removal of the sealant (fig 6)

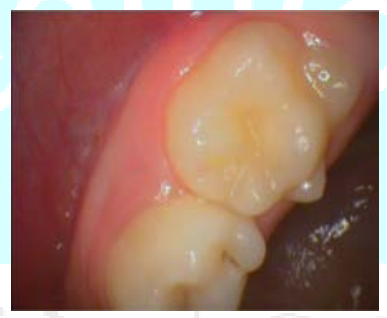

Fig 6- R.V. 6-years old. Visual diagnosis after sealant removal.

\section{Discussion}

In this clinical case the visual diagnosis was reinforced by laser-fluorescence with VistaCam iX proof. The visual classification system International Caries Detection and Assessment System (ICDAS-II) was developed as a method for standardized data collection in different settings and better comparison between studies. Reproducibility and accuracy of ICDAS-II have already shown to be promising for occlusal caries detection (1,3). ICDAS-II is an accessible set of criteria for dentists, researchers and professors and presents acceptable sensitivity and specificity to the detection of occlusal caries $(4,5,6,7)$.

The results from the LF diagnosis made the defect in the sealant more outstanding, however the result from the caries detection was false positive, contrary to the result from the visual diagnostics, by which the defect in the sealant was marked, but no caries was detected. The VistaProof fluorescence camera and the recently devised VistaCam iX (both: D"urr Dental, Bietigheim- Bissingen, Germany) have LEDs that emit high-energy blueviolet light at $405 \mathrm{~nm}$ onto the tooth surface. This wavelength stimulates porphyrins produced by caries-related bacteria to emit red light, containing less energy. Sound enamel, in contrast, sends out green light. This fluorescence is recorded by the camera, transferred to a computer, and processed with special software (DBSWIN,D"urr). The result is a digital image that shows lesions in different colors with respective numerical values between 0 and 4 , predicting the extent and depth of caries $(1,2,8,9,10)$. The influence of sealants on LF measurements has been examined in several studies. Accordingly, in these studies, conflicting results were obtained $(11,12,13,14,15)$. In most of these studies Diagnodent was used for the LF diagnostics. However the basic work principles of these diagnostic tools are the same. As it was already mentioned, there was a variety of outcomes in LF measurements after the 
application of sealants. These differences could be due to differences in the used methods for surface cleaning, type of the sealant, etching time $(11,13,16)$. In some of the studies LF readings after application of opaque and clear sealants have been compared. Most of them, such as studies by Krause et al. (14), concluded that opaque sealants decrease the results from LF readings. On the other side, in a study performed by Bahrololoomi Z.a, Khodabakhsh M.b, Khaksar Y.c in 2014 in Iran on Helioseal sealants, LF measurements tended to increase after sealing with both clear and opaque sealants, but it was statistically significant only in clear sealant group (11).

A study conducted in the University of California by Holtzman $\mathrm{J}$ et al. assessed early occlusal caries preand post- silanization with clear and opaque sealant using several methods, among which visual diagnosis using ICDAS II and LF diagnosis using Diagnodent. ICDAS-II and LF assessments pre- and post- sealant were compared with the gold standard (histopathology). In the study the sensitivity and specificity of both methods were estimated. ICDAS II visual diagnosis method demonstrated high sensitivity (92.3\%) and specificity (83.3\%) before silanization, while LF demonstrated low sensitivity (65.2\%) but high specificity (97.6\%). Re- examination with ICDAS-II post-sealant application was not conducted, but histological analysis was performed. However, in the presence of sealants, LF assessment of caries severity demonstrated high sensitivity (95.1\%), but extremely low specificity (10.3\%). The presence of either dental sealant on the occlusal surface greatly improved LF SE, but dramatically reduced SP (15).

The most common opacifying filler in sealants is TiO2. This filler facilitates the application of sealant and its visual assessment in recall visits (12). As the TiO2 content increases, fluorescence transmission is attenuated; this indicates that the existing $\mathrm{TiO} 2$ in sealants might interfere with the fluorescence transmitted from caries or laser devices. The existing fillers and $\mathrm{TiO} 2$ in sealants create intrinsic fluorescence and the scattering of laser beam. Consequently, they can cause false positive and false negative results. Furthermore, other components of different sealants might affect LF readings. Some of the studies report a significant increase in LF readings after clear sealant application (13). While others, like Diniz et al. and Krause et al. concluded that the effect of clear sealants on LF measurements is not statistically significant (14). However, in the database that we search, we didn't find any similar studies, including the use of VistaCam iX proof, and the effect of different sealing materials on it.

\section{Conclusion}

In conclusion, VistaCam is a reliable, helpful diagnostic method, a useful addition to visual diagnostics on the ICDAS system. Further studies should be performed with different types of sealants as clinical trials to address the influence of pit and fissure sealants on LF measurements more precisely.

\section{References}

1. Jablonski-Momeni A., Stucke J.,Steinberg T., Heinzel-Gutenbrunner M. „Use of ICDAS-II, Fluorescence-BasedMethods, and Radiography in Detection and Treatment Decision of Occlusal Caries Lesions: An In Vitro Study". International Journal of Dentistry, 2012 Article ID 371595.

2. Guerra F., Corridore D., Mazur M. et al. /Senses Sci 2016; 3 (4):317-322 doi: 10.14616/sands2016 - 4-317322 
3. N. Pitts, "ICDAS - an international system for caries detection and assessment being developed to facilitate caries epidemiology, research and appropriate clinical management," Community Dental Health, vol. 21, no. 3, pp. 193-198, 2004.

4. Presoto, Trevisan, Andrade et al. "Clinical effectiveness of fluorescence, digital images and ICDAS for detecting occlusal caries "; Rev Odontol UNESP. 2017 Mar-Apr; 46(2): 109-115

5. Jablonski-Momeni A, Heinzel-Gutenbrunner M, Klein SM. In vivo performance of the VistaProof fluorescence-based camera for detection of occlusal lesions. Clin Oral Investig. 2014 Sep;18(7):1757-1762

6. Jablonski-Momeni A, Heinzel-Gutenbrunner M, Vill G. Use of a fluorescence-based camera for monitoring occlusal surfaces of primary and permanent teeth. Int J Paediatr Dent. 2016 Nov;26(6):448-456.

7. Qudeimat MA, Alomari QD, Altarakemah Y, Alshawaf N, Honkala EJ. Variables affecting the inter- and intra-examiner reliability of ICDAS for occlusal caries diagnosis in permanent molars. J Public Health Dent. 2016;76(1):9-16.

8. Jablonski-Momeni A., F. Liebegall, R. Stoll, M. Heinzel-Gutenbrunner, and K. Pieper, "Performance of a new fluorescence camera for detection of occlusal caries in vitro," Lasers in Medical Science. $2013 ; 28(1): 101-109$.

9. Guerra F, Mazur M, Rinaldo F, et al." New diagnostic technology and hidden pits and fissures caries"Senses Sci 2015; 2 (1):20 -23

10. Guerra F., Corridore D., Mazur M. et al. „Early caries detection: comparison of two procedures. A pilot study";Senses Sci 2016; 3 (4):317-322

11. Bahrololoomi Z., Khodabakhsh M., Khaksar Y. The Effects of Opaque and Clear Pit and Fissure Sealants on Infrared LaserFluorescence Measurements. J Dent Shiraz Univ Med Sci., June 2014; 15(2): 63-67.

12. Gostanian HV, Shey Z, Kasinathan C, Caceda J, Janal MN. An in vitro evaluation of the effect of sealant characteristics on laser fluorescence for caries detection. Pediatr Dent 2006; 28: 445450.

13. Askaroglou E, Kavvadia K, Lagouvardos P, Papagiannoulis L. Effect of sealants on laser fluorescence caries detection in primary teeth. Lasers Med Sci 2011; 26: 29-34.

14. Krause F, Braun A, Frentzen M, Jepsen S. Effects of composite fissure sealants on IR laser fluorescence measurements. Lasers Med Sci 2008; 23: 133-139.

15. Holtzman J., Ballantine J., Fontana M. et al. "Assessment of early occlusal caries pre- and postsealant application - an imaging approach" Lasers Surg Med. 2014 August ; 46(6): 499-507

16. Guerra F, Mazur M, Rinaldo F. et al." Clinical procedure in sealing pit and fissure using technological aids: VistaCam iX Proof and Combi“ Senses Sci 2016; 3 (1):157-162

\section{Corresponding author:}

\section{Lilyana Sheteva,}

Assistant professor in the Department of Pediatric Dentistry,

Faculty of Dental Medicine,

Medical University - Plovdiv,

Hristo Botev 3, Plovdiv, Bulgaria,

email: shtereva.lilyana@abv.bg

tel: 0893543545; 